\title{
An Efficient Dimensionality Reduction Method for the Classification of Satellite Remote Sensing Hyperspectral Images
}

\author{
Md. Rashedul Islam \\ Department of Computer Science \\ and Engineering \\ Hajee Mohammad Danesh Science \\ and Technology University, \\ Bangladesh
}

\author{
Ayasha Siddiqa \\ Department of Computer Science \\ and Engineering \\ Hajee Mohammad Danesh Science \\ and Technology University, \\ Bangladesh
}

\author{
Nafisa Tasnim \\ Department of Computer Science \\ and Engineering \\ Hajee Mohammad Danesh Science \\ and Technology University, \\ Bangladesh
}

\begin{abstract}
Finding an informative subset of features from the original hyperspectral images has become essential because of its wide applications in ground object identification. However, information extraction from hyperspectral images is becoming challenging because of its high correlation among the image bands and spectral and spatial redundancy. This paper proposed a feature reduction approach, combining both feature extraction and feature selection. A combination of Minimum Noise Fraction (MNF) and information-based measure, cross cumulative residual entropy (CCRE), is proposed to select the subset of features from the original image to obtain improved classification accuracy. In the proposed method, feature ranking is improved by scaling the CCRE to a specific range to avoid redundant features. The proposed technique (MNF-nCCRE) is tested on two hyperspectral images captured by the NASA AVIRIS sensor and HYDICE sensor. The experimental results typically indicate a noticeable improvement in terms of classification accuracy. The proposed technique shows $96.8 \%$, and $99.10 \%$ classification accuracy on AVIRIS and HYDICE hyperspectral data, respectively, higher than the standard approaches studied.
\end{abstract}

\section{General Terms}

Remote sensing image classification, Feature Selection, Feature mining, Data Mining.

\section{Keywords}

Feature extraction, subspace identification, minimum noise fraction, AVIRIS, HYDICE, hyperspectral images classification.

\section{INTRODUCTION}

Hyperspectral data cube contains hundreds of image bands with a fine spectral resolution, e.g., $0.01 \mu \mathrm{m}$, covering visible light to a near-infrared range of the frequency spectrum [1]. Each of these image bands is termed as individual features as they contain different intensities for each of the ground objects [1]. Therefore, hyperspectral image isapowerful source of information for the detection of ground objects. A hyperspectral image is a three-dimensional data cube. For image processing tasks such as classification faces some difficulties, such as the input image bands of hyperspectral datacube are highly correlated. Moreover, all the bands are not equally important for a specific application [2]. Since the hyperspectral sensor continuously captures images, some image bands contain less discriminatory information about the ground objects [3]. Another critical challenge is the classification of this high-dimensional data cube since enough training samples are not available. For instance, if the ratio of the training samples and the number of input images becomes very small, the classification accuracy of the test samples starts to decrease gradually, and this effect is termed as 'Hughes phenomena' or curse of dimensionality [4]. Therefore, it is essential to reduce the high dimensional data to relevant subspace to improve classification accuracy. Thus, an effective technique is a concern in this paper.

Different feature reduction approaches can be applied to extract informative features from hyperspectral images effectively. Feature reduction can be achieved in both feature extraction and feature selection. Feature extraction maps the input images to a new space $\mathrm{K}$ from original space $\mathrm{N}$ where $\mathrm{K}$ $<\mathrm{N}$ through linear or nonlinear transformation. The most commonly used unsupervised feature extraction method is Principal Component Analysis (PCA) [5-6]. Although PCA is based on the fact that neighboring bands are highly correlated and used to transform the original highdimensional data to lower dimension by removing the correlation among the bands using the higher variance, it does not consider the noise factor of an image. The variance of hyperspectral images did not reflect the actual signal-to-noise ratio (SNR) [7]. Therefore, slight variance does not mean poor image quality; higher variance may have lower SNR than other bands [8].

Although PCA has been successfully applied in many remote sensing data, it is not scale-invariant, is quite variable with respect to the information content of a particular image, and does not guarantee good class separation in the transformed space [9-10]. Hence, minimum noise fraction (MNF) is proposed as the better technique for feature extraction as it can minimize the disadvantages of PCA depending on image quality. In MNF, the components are arranged in terms of signal-to-noise ratio, no matter how noise is distributed in spectral bands [7]. Although feature extraction transforms the original extensive data to a new space with few features, ranking the new features is the primary concern shown in some research [1-2], [11-13]. Since MNF is an unsupervised technique that solely considers SNR, some of the classes may affect the classification accuracy thatis not present in the first few features. Therefore, only MNF is not the effective way for dimensional reduction. So, feature selection is applied for effective feature reduction.

Cross Cumulative Residual Entropy (CCRE) is a popular supervised feature selection method. Itcan measure both the 
linear and nonlinear relationships between the spectral bands and the target classes,making it suitable for effective subspace detection [2], [14-16]. But it is challenging to rank features based solely on CCRE between two variables because the CCRE does not have any range. Therefore, a comparison of two CCRE values may not always be preferable. Accordingly, this paper scales the CCRE value to a specific range, i.e., 0 to 1 , to compare two values [17-18]. Thus, an improved subspace detection technique is proposed using a normalized CCRE (nCCRE) over the new generated features. This new space can maximize the relevancy and minimizing the redundancy of the selected features.

The main contributions of this paper are as follows.

i. A hybrid feature reduction method has been proposed to ensure both spectral and spatial attributes, including feature extraction and selection.

ii. A normalized CCRE information-based feature selection is applied to improve the quality of the chosen features by utilizing the max-relevancy minredundancy measure.

The rest of the paper is organized as follows. Section 2describes the basic algorithm of MNF and CCRE. Then, the proposed feature extraction, called MNF-nCCRE,improves the quality of the chosen features. Section 3 intricately explains the conducted experiments on the two real HSI datasets using the proposed feature reduction approach with state-of-the-art. At last, Section 4 summarizes the outcomes and concludes the paper.

\section{METHODOLOGY}

The proposed feature reduction method encompasses two main steps: (i) Minimum Noise Fraction based feature extractionand (ii) feature selection using normalized CCRE based on max-relevancy min-redundancy measures on the transformed features.

\subsection{Minimum Noise Fraction}

MNF can estimate the inherent feature dimension of image availability, and its existence is a superposition of two PCA. The MNF is suitable as it selects the signal-to-noise ratio rather than the global variance to measure relevant features [7].

Suppose the input hyperspectral image is denoted as $\mathrm{X}$, where $\mathrm{X}=[\mathrm{x} 1, \mathrm{x} 2 \ldots \ldots \mathrm{xp}] \mathrm{T}$. Here, $\mathrm{p}$ is the number of spectral bands. If noise is present in the signal, $\mathrm{X}=\mathrm{S}+\mathrm{N}$, where $\mathrm{S}$ and $\mathrm{N}$ are the signal component and noise component in images. The signal covariance matrix can be expressed as

$$
\mathrm{C}(\mathrm{X})=\Sigma=\Sigma \mathrm{S}+\Sigma \mathrm{N}
$$

Where $\Sigma \mathrm{S}$ and $\Sigma \mathrm{N}$ is the covariance of the signal and noise, respectively, using the ratio of noise, the linear transformation MNF can be defined as:

$$
\mathrm{Y}=\mathrm{A}^{\mathrm{T}} \mathrm{X}
$$

Where the matrix $\mathrm{A}$ is the eigenvector matrix of

$$
\Sigma_{-1} \Sigma_{\mathrm{N}}=\Lambda \mathrm{A} \text {. }
$$

The diagonal matrix $\Lambda$ is an eigenvalues matrix. The noise ratio of the corresponding component of $\Lambda$ is

$$
\frac{\operatorname{Var}\left(a_{i}^{T} \mathbf{N}\right)}{\operatorname{Var}\left(a_{i}^{T} \mathbf{X}\right)}=\frac{a_{i}^{T} \sum_{\mathbf{N}} a_{i}}{a_{i}^{T} \sum_{\mathbf{X}} a_{i}}
$$

Where $\operatorname{Var}\{\}$ calculates the variance and ai is the ith component of the eigenvector matrix A.

The hyperspectral images are arranged in accordance with higher SNR through the corresponding MNF transformation. The first few components contain the less noisy information of the image, and the decreasing component contains more noises in information, unlike PCA, which arrange with global variance.

\subsection{Cross Cumulative Residual Entropy}

The Cross Cumulative Residual Entropy (CCRE) is a popular similarity measure tool [19]. CCRE can be used to measure the similarity of two images in which cumulative residual distribution is used instead of probabilistic distribution [20]. The CCRE of two images I and $\mathrm{J}$ is given by

$$
C_{C C R E}=\sum_{u=1}^{L} \sum_{v=1}^{L} G(u, v) \log \left(\frac{G(u, v)}{G_{I}(u) P_{J}(v)}\right)
$$

where $L$ represents the most significantpixelvalueoftheimages, $\mathrm{G}(\mathrm{u})$ is the joint cumulative residual distribution, $\mathrm{GI}(\mathrm{u})$ is the marginal cumulative residual distribution of $\mathrm{I}$, and $\mathrm{PJ}(\mathrm{v})$ is the marginal probability of $\mathrm{J}$.

\subsection{Proposed Algorithm (MNF-nCCRE).}

CCRE has been measured between the new features generated from $\operatorname{MNF}\left(\mathbf{Y}_{\mathrm{i}}\right)$ and the available training class labels $\mathrm{C}$ to select the subset of relevant features. Thus, the most informative feature is calculated as [21]

$$
V=\operatorname{Max}_{i \in p} C_{C C R E}\left(\mathbf{Y}_{i}, \mathbf{C}\right),
$$

where $\mathrm{V}$ represents the first feature, selected for classification and assigned to $\mathrm{S}$. In this way, one can sort the MNF components, and the first few components may be the informative feature for classification.However, the selected features using Equation (6) may have some redundancy. The objective is to maximize the relevance and minimize the redundancy among the selected features. Therefore, the next informative feature is selected based on the following model.

$$
G\left(\mathbf{Y}_{i}, k\right)=\left[C_{C C R E}\left(\mathbf{Y}_{i}, \mathbf{C}\right)-\frac{1}{|S|} \sum_{i, j \in S} C_{C C R E}\left(\mathbf{Y}_{i}, \mathbf{Y}_{j}\right)\right], \mathbf{Y}_{i} \not \subset S(7)
$$

However, the CCRE value in the above Equation is not bounded to a specific range. Therefore, the value G(Yi,k) in the above method is difficult to use directly, as it may be affected by the entropy of two variables and not bounded to a specific range. Therefore, the normalized CCRE value can be used in equation (7). The normalized CCRE between MNF component $\mathrm{Y}$ and class label $\mathrm{C}$ can be defined as

$$
C_{C C R E}(\mathbf{Y}, \mathbf{C})=\frac{C_{C C R E}(\mathbf{Y}, \mathbf{C})}{\sqrt{C_{C C R E}(\mathbf{Y}, \mathbf{Y}) C_{C C R E}(\mathbf{C}, \mathbf{C})}}(8)
$$

Here, the method proposed nCCRE, utilizing the normalized CCRE in Equation (8), and as a result, the proposed subsets of the feature method have been defined as

$$
G\left(\mathbf{Y}_{i}, k\right)=\left[C_{C C R E}\left(\mathbf{Y}_{i}, \mathbf{C}\right)-\frac{1}{|S|} \sum_{i, j \in S} C_{C C R E}\left(\mathbf{Y}_{i}, \mathbf{Y}_{j}\right)\right], \mathbf{Y}_{i} \not \subset S \text { (9) }
$$

Following is a summarized algorithm for the proposed feature reduction method. Here, $\mathrm{S}$ represents the set of selected features.

\section{Algorithm 1. MNF-nCCRE}


i. Begin $\{\mathbf{Y}$ : the projected data matrix after applying MNF \}

ii. Initialize the feature subspace to null, $\mathbf{S}_{\mathbf{0}}=\{\Phi\}$

iii. Select the $1^{\text {st }}$ feature, $\mathbf{Y}_{\mathbf{j}}$ using Equation (6) through utilizing Equation (8) and set

$$
S_{1}=S_{0} \cup Y_{j}
$$

iv. $\quad$ For selecting the remaining features, do

v. Utilize Equation (9) and update $\mathbf{S}$

vi. OutputS as the subspace of informative features

Output the set $\mathbf{S}$, containing the selected features.

\section{EXPERIMENTS}

\subsection{Remote Sensing Datasets}

For the experiment presented here, two real hyperspectral image data sets were used. Data set 1 consists of 220 bands collected by AVIRIS sensor over the Indian Pines test site in the USA, having $145 \times 145$ spatial resolutions. Sixteen classes are defined in the ground truth map [22]. The insufficient training data "Grass/Pasture mowed" and "Oats" for the AVIRIS image was not used in the experiment. Data set 2 consisted of 191 channels with $1280 \times 307$ pixels and was collected by the HYDICE sensor over the Washington DC MALL in 1995 [23]. For the HYDICE data, "paths" were not used as they have insufficient training samples. Table 1 and 2 represent the training and testing samples for AVIRIS and HYDICE, respectively, and were used in the experimental procedure. For the classification, both the training and testing samples are selected based on the groundtruth of the original image shown in Fig. 1. and Fig. 2.

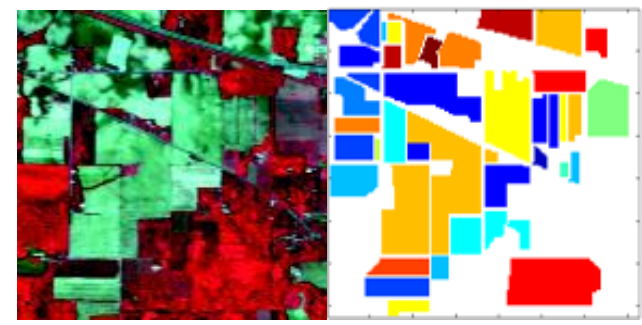

(a)(b)

Fig. 1.AVIRIS HSI Data(a) False color RGB image $\operatorname{RGB}(50,27,17)(b)$ Ground truth image.

\subsection{Experimental Setting}

The kernel support vector machine (SVM) classifier with RBF kernel is used for classification accuracy measurement. The classifier has been trained using 10-fold cross-validation to select the best cost parameter $\mathrm{C}$ and kernel width $\gamma$ [24]. The kernel parameters $(\mathrm{C}=8$ and $\gamma=2.7)$ for AVIRIS and $(\mathrm{C}=2$ and $\gamma=1.8$ ) for HYDICE were selected for classification using 12 features for AVIRS data and eight features for HYDICE data.

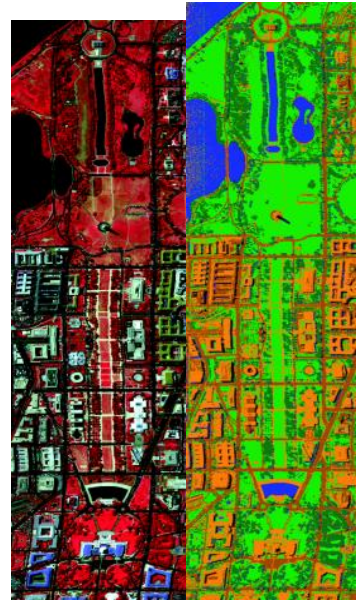

(a)(b)

Fig. 2.HYDICE HSI data (a) False-color RGB image RGB(50,52,36)(b) Ground truth image.

Table 1.Training and Testing samples of AVIRIS data

\begin{tabular}{|c|c|c|}
\hline Class Name & Training samples & Testing samples \\
\hline Alfalfa & 16 & 16 \\
\hline Wheat & 43 & 65 \\
\hline Bldge-Grass & 10 & 22 \\
\hline Soybean-min & 131 & 166 \\
\hline Stone-Steel & 20 & 40 \\
\hline Soybean-notil & 150 & 160 \\
\hline Grass/Pasture & 70 & 150 \\
\hline Corn-notill & 47 & 100 \\
\hline Soybean Clean & 20 & 50 \\
\hline Corn-min & 65 & 102 \\
\hline Hay-windrowed & 150 & 166 \\
\hline Woods & 279 & 290 \\
\hline Grass/Trees & 30 & 150 \\
\hline Corn & 66 & 70 \\
\hline Total & 1097 & 1547 \\
\hline
\end{tabular}

Table 2.Training and testing samples of HYDICE data

\begin{tabular}{|c|c|c|}
\hline Class Name & Training Samples & Testing Samples \\
\hline Shadow & 20 & 16 \\
\hline Tree & 367 & 1206 \\
\hline Roof & 117 & 108 \\
\hline Water & 425 & 580 \\
\hline Street & 288 & 465 \\
\hline Grass & 200 & 850 \\
\hline Total & 1417 & 3225 \\
\hline
\end{tabular}

\subsection{Feature Extraction Results}

In this analysis, new features are generated through MNF. Then the feature selection is performed on the new generated features based on the normalized CCRE (MNF-nCCRE). The proposed method is compared with standardtechniques such as PCA, MNF, CCRE, and MNF-CCRE. For each process, the order of the selected features is listed in table 3. It can be seen that the proposed MNF-nCCRE select the MNF component-2 as the first ranked feature as MNF component-1 is the noisy feature. Fig. 3 visually shows that the MNF component- 1 is noisy as compared to MNF component-2.

Fig. 3 visually shows the benefits of applying nCCRE over traditional MNF images of the MNF component of 1 and MNF component of 2 of the AVIRIS data. 
Table 3.Selected Features for Classification

\begin{tabular}{|l|l|l|}
\hline Data Set & Methods & Orders of selected features \\
\hline \multirow{5}{*}{ AVIRIS } & PCA & PC: $1,2,3,4,5,6,7,8,9,10,11,12$ \\
\cline { 2 - 3 } & MNF & MNF-C: $1,2,3,4,5,6,7,8,9,10,11,12$ \\
\cline { 2 - 3 } & CCRE & $\begin{array}{l}\text { Bands: } 22,12,29,28,67,24,26,30,31, \\
19,110,38\end{array}$ \\
\cline { 2 - 3 } & MNF-CCRE & $\begin{array}{l}\text { MNF-C: } 2,3,4,6,5,7,11,12,13,14,10, \\
9\end{array}$ \\
\cline { 2 - 3 } & MNF-nCCRE & $\begin{array}{l}\text { MNF-C: } 2,4,5,6,8,9,10,12,15,7,11, \\
13\end{array}$ \\
\hline \multirow{5}{*}{ HYDICE } & PCA & PC: $1,2,3,4,5,6,7,8$ \\
\cline { 2 - 3 } & MNF & MNF-C: $1,2,3,4,5,6,7,8$ \\
\cline { 2 - 3 } & CCRE & Bands: $84,101,51,77,28,57,163,165$ \\
\cline { 2 - 3 } & MNF-CCRE & MNF-C: $2,4,6,3,7,11,12,19$ \\
\cline { 2 - 3 } & MNF-nCCRE & MNF-C: $2,3,5,3,6,10,11,8$ \\
\hline
\end{tabular}
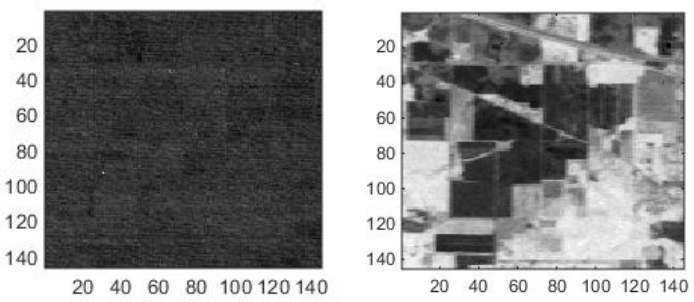

(a)(b)

Fig. 3.Visual representation of (a) MNF component 1 (b) MNF component 2 of AVIRIS data.

Fig. 4 and Fig. 5, Show's the subspace projection of the AVIRIS data indicates that the MNF component-2 is more separable than MNF component-1.

The robustness of the proposed method is also evaluated using feature space analysis. Fig. 7 shows the scatter plots of AVIRIS data using the standard MNF, MNF-CCRE, and the proposed method. For ease, only eight classes have been used in the feature space. From Fig. 4(a) and 4(b), it is seen that many class labels are overlapped with each other. Whereas Fig 4(c) shows, only a few overlapsbetween the class labels,and classes are more separable than the two different approaches. As a result, the advantage of feature selection is apparent over the extracted data, which is more separable.

Fig. 5 also shows the feature space analysis of the conventional MNF, MNF-CCRE, and the proposed methods of the HYDICE data. The result also demonstrates that the proposed feature reduction approach can separate the classes efficiently than the studied techniques.

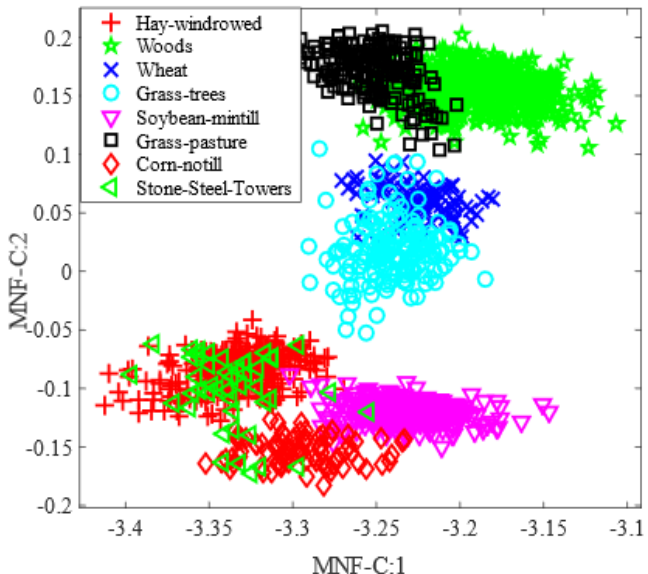

(a)

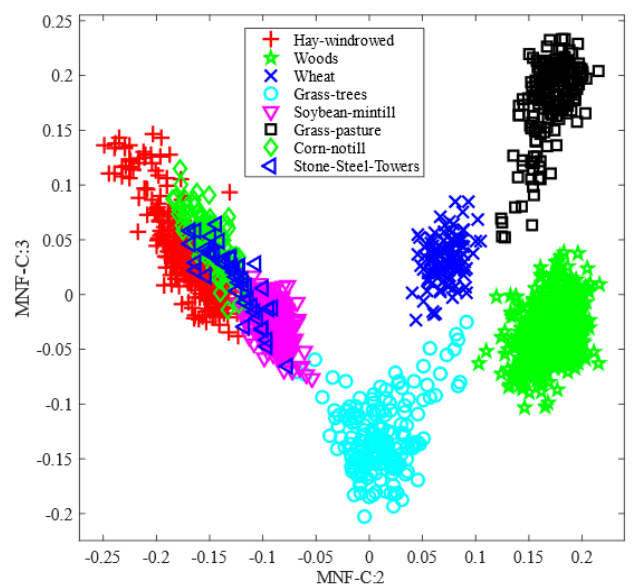

(b)

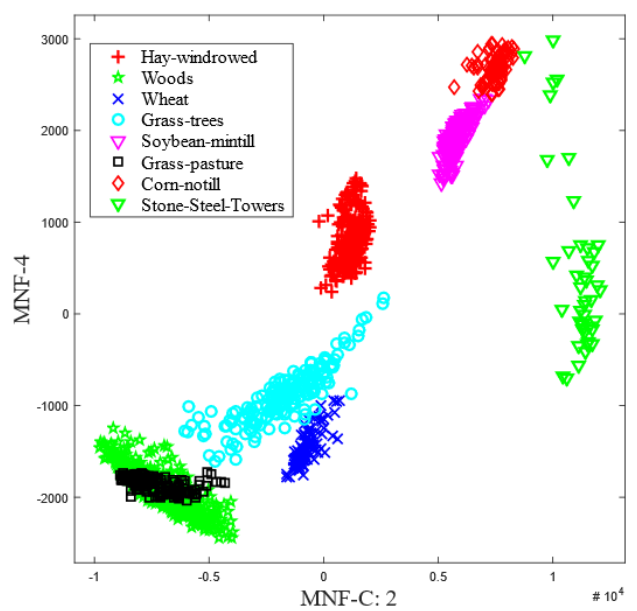

(c)

Fig. 4.Scatter plots of two ranked features of (a) MNF,(b) MNF-CCRE, and (c) MNF-nCCRE methodfor AVIRIS data. 


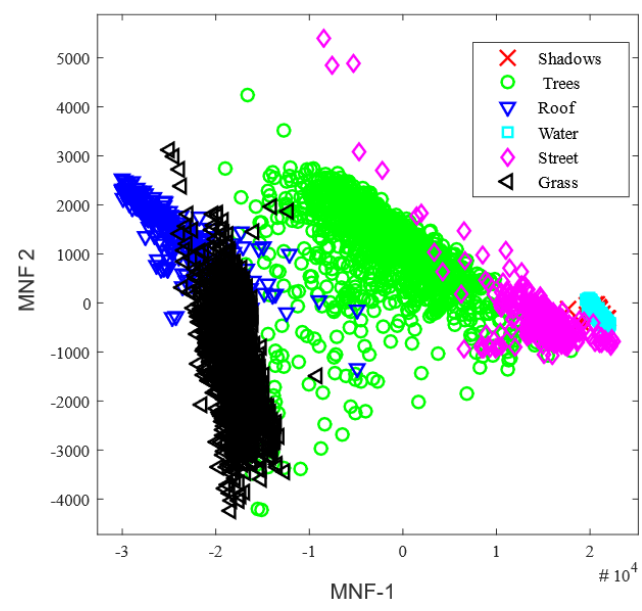

(a)

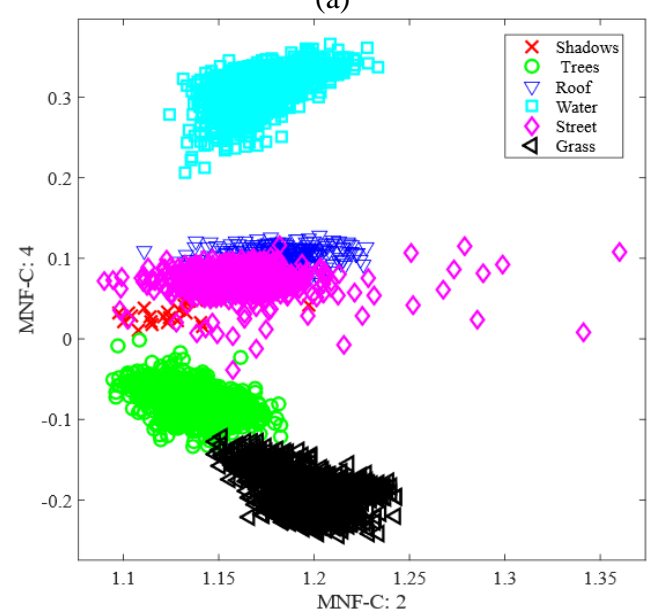

(b)

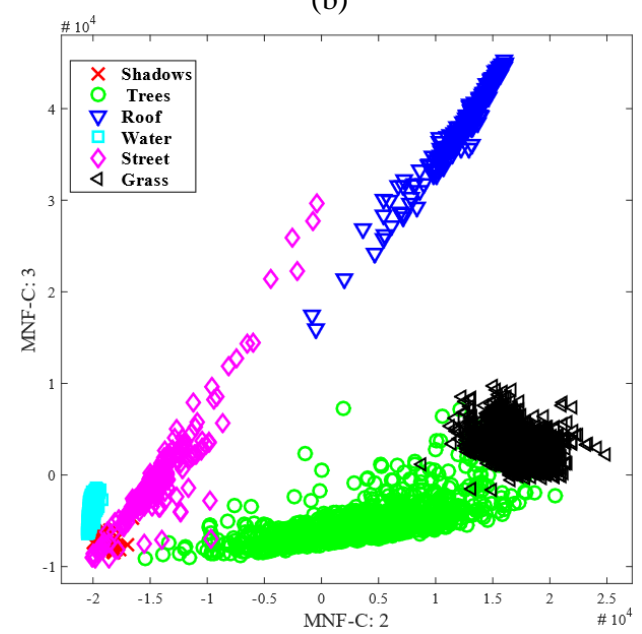

(c)

Fig. 5.Scatter plots of two ranked features of (a) MNF,(b) MNF-CCRE, and (c) MNF-nCCRE method for HYDICE data

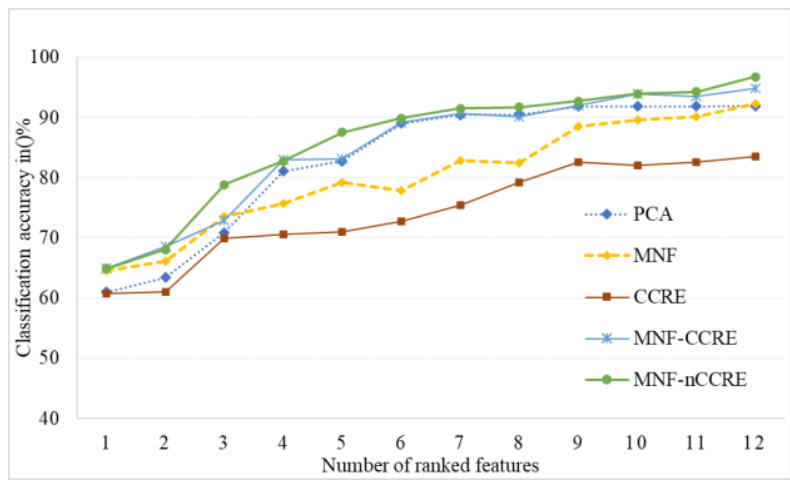

Fig. 6.Classification accuracy plot in \% of AVIRIS data

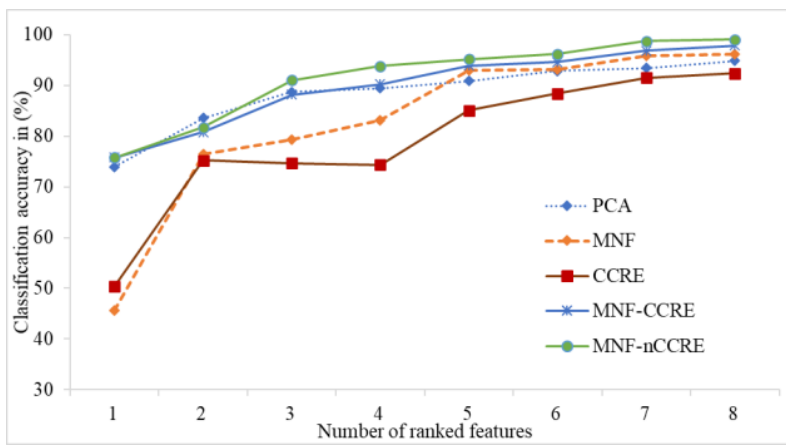

Fig. 7.Classification accuracy plot in \% of HYDICE data

\subsection{ClassificationResults}

The achievement of the proposed approach is evaluated in terms of classification accuracy. The selected features listed in table 3 are applied for classification with kernel SVM. The proposed method was compared with the popular PCA, MNF, CCRE, and MNF-CCRE approaches. For each method and dataset, the overall classification accuracies of AVIRIS and HYDICE data sets are given in Fig. 6 and Fig. 7, respectively, with respect to the order of selected features. The classification accuracy for the original input data AVIRIS (without feature selection/feature extraction) is $71.23 \%$, encouraging feature reduction. The classification accuracy of conventional PCA, MNF, CCRE, and MNF-CCRE with the first 12 features is $91.9 \%, 92.29 \%, 83.6 \%$, and $94.8 \%$, respectively. The proposed method shows the classification accuracy of $96.8 \%$ for AVIRIS data, which is higher than the othermethods studied. For the HYDICE data, the conventional PCA, MNF, CCRE, and MNF-CCRE with eight features show the classification accuracy of $94.88 \%, 96.12 \%, 92.40 \%$, and $97.86 \%$,respectively. The proposed method shows the classification accuracy of $99.10 \%$ with eightselected features, which higher than the other methods studied. Table 4 and Table 5 are listed the classification accuracy of PCA, MNF, CCRE, MNF-CCRE, and proposed MNF-nCCRE. The other performance evaluation metrics, such as average accuracy (AA), kappa, and F1 score, are also evaluated and finds outperform compared to the method studied.

Table 4.Classification results of AVIRIS data

\begin{tabular}{|c|c|c|c|c|c|}
\hline Class & PCA & MNF & CCRE & $\begin{array}{c}\text { MNF- } \\
\text { CCRE }\end{array}$ & $\begin{array}{c}\text { MNF- } \\
\text { nCCRE }\end{array}$ \\
\hline Alfalfa & 88.24 & 87.50 & 87.50 & 88.24 & 88.24 \\
\hline Wheat & 90.91 & 92.31 & 86.96 & 92.86 & 92.86 \\
\hline Bldge-Grass & 88.00 & 88.00 & 81.48 & 91.67 & 100.00 \\
\hline Soybean-min & 94.48 & 97.87 & 92.31 & 98.59 & 98.77 \\
\hline
\end{tabular}




\begin{tabular}{|c|c|c|c|c|c|}
\hline Stone-Steel & 97.22 & 97.22 & 90.91 & 97.22 & 97.22 \\
\hline Soybean-notil & 90.54 & 93.10 & 83.33 & 93.33 & 98.59 \\
\hline Grass/Pasture & 87.42 & 87.50 & 80.00 & 93.33 & 93.75 \\
\hline Corn-notill & 95.19 & 95.24 & 94.12 & 97.09 & 97.09 \\
\hline Soybean Clean & 81.82 & 88.24 & 70.00 & 88.24 & 88.24 \\
\hline Corn-min & 98.00 & 98.04 & 89.11 & 98.04 & 98.04 \\
\hline Hay-windrowed & 91.19 & 91.72 & 80.46 & 94.12 & 100.00 \\
\hline Woods & 92.72 & 92.72 & 80.13 & 96.67 & 100.00 \\
\hline Grass/Trees & 94.87 & 95.48 & 86.55 & 95.54 & 95.54 \\
\hline Corn & 86.67 & 86.67 & 76.47 & 86.67 & 86.67 \\
\hline AA & 91.23 & 92.26 & 84.24 & 93.69 & 95.36 \\
\hline OA & 91.9 & 92.29 & 83.6 & 94.8 & $\mathbf{9 6 . 8}$ \\
\hline KAPPA & 91 & 92.21 & 81.8 & 94.2 & $\mathbf{9 6 . 4}$ \\
\hline F1 Score & 91.79 & 92.47 & 84.35 & 94.12 & $\mathbf{9 5 . 6 2}$ \\
\hline
\end{tabular}

Table 5.Classification results of HYDICE data

\begin{tabular}{|c|c|c|c|c|c|}
\hline Class & PCA & MNF & CCRE & $\begin{array}{c}\text { MNF- } \\
\text { CCRE }\end{array}$ & $\begin{array}{c}\text { MNF- } \\
\text { nCCRE }\end{array}$ \\
\hline Shadow & 88.24 & 100.00 & 88.24 & 100.00 & 100.00 \\
\hline Tree & 94.88 & 96.88 & 94.66 & 97.00 & 99.83 \\
\hline Roof & 98.90 & 98.90 & 98.90 & 99.01 & 99.01 \\
\hline Water & 96.60 & 97.22 & 96.47 & 97.22 & 98.11 \\
\hline Street & 87.35 & 89.46 & 87.09 & 98.47 & 98.50 \\
\hline Grass & 97.88 & 97.89 & 89.44 & 99.05 & 99.07 \\
\hline AA & 93.97 & 96.73 & 92.47 & 98.46 & 99.09 \\
\hline OA & 94.88 & 96.12 & 92.40 & 97.86 & $\mathbf{9 9 . 1 0}$ \\
\hline Kappa & 93.05 & 94.74 & 89.69 & 97.09 & $\mathbf{9 8 . 7 8}$ \\
\hline F1 Score & 93.35 & 95.34 & 91.78 & 97.82 & $\mathbf{9 8 . 6 4}$ \\
\hline
\end{tabular}

\section{CONCLUSION}

The HSI is a high-dimensional and voluminous data cube. Therefore, dimension reduction is necessary for such a large dataset. This paper proposed a combination of feature extraction (MNF) and feature selection (nCCRE) for dimensionality reduction. Feature selection over feature extraction improves the quality of the output features from traditional MNF. This is because MNF-nCCRE finds the subsets less noisyand provides relevant information about the desired ground objects. The improvement in accuracies shows the suitability of the proposed method. The proposed MNFnCCRE is also capable of giving better results when only a few training samples are available.

\section{REFERENCES}

[1] X. Jia, B. Kua, and M. M. Crawford, "Feature Mining for Hyperspectral Image Classification," Proceedings of the IEEE, vol. 101, no. 3, pp. 676-679, 2013.

[2] M. R. Islam, B. Ahmed, \& M. A. Hossain, "Feature reduction based on segmented principal component analysis for hyperspectral images classification," International Conference on Electrical, Computer and Communication Engineering (ECCE), 2019.

[3] B. Guo, S. R. Gunn, R. I. Damper and J. D. B. Nelson, "Band Selection for Hyperspectral Image Classification Using Mutual Information," IEEE Geosci. Remote Sens. Lett., vol. 3, no. 4, pp. 522-526, Oct. 2006.

[4] G. Hughes, "On the mean accuracy of statistical pattern recognizers," IEEE Trans. Inf. Theory, vol. 14, no. 1, pp. 55-63, Jan. 1968.

[5] L. Ying, G. Yanfeng, and Z. Ye, "Hyperspectral feature extraction using selective PCA based on genetic algorithm with subgroups," in Proc. ICICIC, pp. 652656, 2006.
[6] C. Rodarmel and J. Shan, "Principal Component Analysis for hyperspectral image classification," ACM Surveying and Land Information Science, vol. 62, no. 2, pp. 115-122, 2002.

[7] Green, Berman, M. Switzer, and Craig, "A transformation for ordering multispectral data in terms of image quality with implications for noise removal," IEEE Trans. Geosci. Remote Sens., vol. 26 no. 1, pp. 6574, Jan, 1988.

[8] C. Chang, Qian Du, "Interference and Noise-Adjusted Principal Components Analysis," IEEE Trans. Geosci. Remote Sens., vol. 37, no. 5, pp. 2387-2396, Sept. 1999.

[9] L. Gao, B. Zhao, X. Jia, W. Liao, B. Zhang, “Optimized Kernel Minimum Noise Fraction Transformation for Hyperspectral Image Classification," Remote Sensing. vol. 9, no. 6, pp. 5482017.

[10] G. Luo, G. Chen, L. Tian, K. Qin and S. Qian "Minimum Noise Fraction versus Principal Component Analysis as a Preprocessing Step for Hyperspectral Imagery Denoising," Canadian Journal of Remote Sensing, vol. 42, no. 2, pp. 106-116, 2016.

[11] M. R. Islam, B. Ahmed, \& M. A. Hossain, "Feature reduction of hyperspectral image for classification," Journal of Spatial Science, p. 1-21, 2020.

[12] M. A. Hossain, X. Jia and M. Pickering, "Improved feature selection based on a mutual information measure for hyperspectral image classification," Proc. Int. Geosci. Remote Sens. Symp., pp. 3058-3061, 2012.

[13] M. R. Islam, M.A. Hossain, \& B. Ahmed, "Improved subspace detection based on minimum noise fraction and mutual information for hyperspectral image classification," Proceedings of International Joint Conference on Computational Intelligence, 2020.

[14] J. A. Richards and X. Jia, Remote Sensing Digital Image Analysis, 4th ed. Berlin, Germany: Springer-Verlag, 2006.

[15] C. Qi, Z. Zhou, Q. Wang and L. Hu, "Mutual Information-Based Feature Selection and Ensemble Learning for Classification," 2016 International Conference on Identification, Information and Knowledge in the Internet of Things (IIKI), pp. 116-121, 2016.

[16] Y. Fu, X. Jia, W. Huang and J. Wang, "A comparative analysis of mutual information based feature selection for hyperspectral image classification," 2014 IEEE China Summit \& International Conference on Signal and Information Processing (ChinaSIP), pp. 148-152, 2014.

[17] P. A. Estevez, M. Tesmer, C. A. Perez and J. M. Zurada, "Normalized Mutual Information Feature Selection," in IEEE Transactions on Neural Networks, vol. 20, no. 2, pp. 189-201, Feb. 2009.

[18] A. Siddiqa, M. Ibn Afzal, M. R. Islam, and A. Mahjabin Nitu, "Spectral Subset Detection for Hyperspectral Image Classification," 2019 2nd International Conference on Innovation in Engineering and Technology (ICIET), 2019, pp. 1-6, DOI: 10.1109/ICIET48527.2019.9290596.

[19] Wang, F., \& Vemuri, B. C. (2007). Non-rigid multimodal image registration using cross-cumulative residual entropy. International journal of computer vision, 74(2), 
201-215.

[20] Rao, M., Chen, Y., Vemuri, B. C., \& Wang, F. (2004). Cumulative residual entropy: a new measure of information. IEEE transactions on information theory, 50(6), 1220-1228.

[21] H. Peng, F. Long, and C. Ding, "Feature Selection Based on Mutual Information: Criteria of Max-Dependency, Max-Relevance, and Min-Redundancy," IEEE. Trans. Pattern Recognition and Machine Learning, vol. 28, no. 8, pp. 1226-1238 August 2005.
[22] P. A. Estévez, M. Tesmer, C. A. Perez, and J. M. Zurada, "Normalized mutual information feature selection," IEEE Trans. Neural Netw., vol. 20, no. 2, pp. 189-201, Feb. 2009.

[23] D. A. Landgrebe. [Online]. Available: https://engineering.purdue.edu/ biehl/MultiSpec/hypersp ectral.html

[24] C. Hsu, C. Chang, and C. Lin, "A practical guide to support vector Classification". In: (2003). Appendix: Springer-Author Discount. 